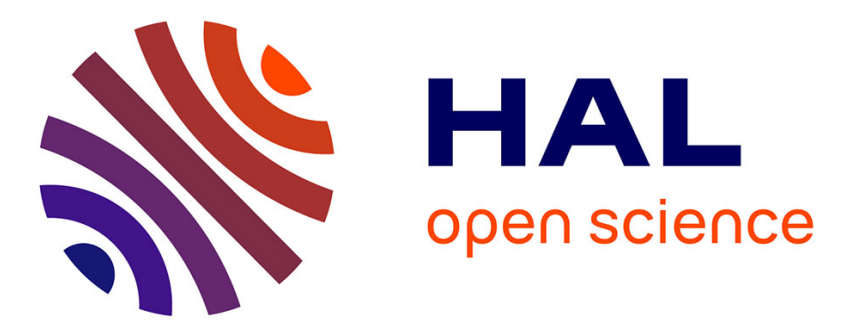

\title{
Arthrose destructrice rapide ou rapidement progressive
} G. Chalès, F. Robin, G. Coiffier

\section{To cite this version:}

G. Chalès, F. Robin, G. Coiffier. Arthrose destructrice rapide ou rapidement progressive. Revue du Rhumatisme monographies, 2021, 88 (2), pp.114-122. 10.1016/j.monrhu.2021.01.005 . hal-03164564

\section{HAL Id: hal-03164564 https://hal.science/hal-03164564}

Submitted on 29 Mar 2021

HAL is a multi-disciplinary open access archive for the deposit and dissemination of scientific research documents, whether they are published or not. The documents may come from teaching and research institutions in France or abroad, or from public or private research centers.
L'archive ouverte pluridisciplinaire HAL, est destinée au dépôt et à la diffusion de documents scientifiques de niveau recherche, publiés ou non, émanant des établissements d'enseignement et de recherche français ou étrangers, des laboratoires publics ou privés. 


\section{Arthrose destructrice rapide ou rapidement progressive \\ Rapidly progressive destructive osteoarthritis}

Gérard Chalès ${ }^{c}$, François Robin ad, Guillaume Coiffier ${ }^{\text {bd }}$
a)
b) Service de Rhumatologie, Hôpital sud, 16 Boulevard de Bulgarie 35203 Rennes Cedex 2, France

c) Service de Rhumatologie, GHT Rance-Emeraude, $\mathrm{CH}$ Dinan, 74 boulevard de Chateaubriand, 22100 Dinan, France.

d) Faculté de médecine, 2 avenue du professeur Léon Bernard - CS 3431735043 Rennes Cedex

e) Inserm, UMR 1241, Institut Nutrition métabolismes et cancer (NuMeCan), CHU de Rennes, Université de Rennes, Rennes, France

\section{CORRESPONDANCE}

Pr G. Chalès, Faculté de médecine, 2 avenue du professeur Léon Bernard - CS 34317 35043 Rennes Cedex

courriel : gerard.chales@orange.fr (06 866710 10) 


\section{Résumé}

L'arthrose destructrice rapidement progressive (ADRP) est considérée comme une entité rare et méconnue. L'ADRP est une arthropathie destructrice qui survient plus souvent chez la femme âgée. Les signes d'imagerie les plus typiques sont le pincement de l'interligne articulaire (chondrolyse), l'existence d'une fracture souschondrale, de géodes de la tête fémorale et de l'acétabulum, l'absence d'ostéophytes, l'aplatissement de la tête fémorale, et des signes d'épanchement. L'ADRP a d'abord été décrite à la hanche mais peut toucher aussi l'épaule et le genou. La pathogénie de l'ADRP n'est pas bien connue, mais la fracture sous-chondrale joue probablement un rôle dans la destruction ostéoarticulaire. Néanmoins, le diagnostic précoce des patients avec une ADRP de la hanche est important pour essayer de réduire la complexité de l'acte chirurgical.

Mots-clés arthrose destructrice rapide, hanche, épaule, genou, IRM, chondrolyse, fracture sous-chondrale, pathogénie

\section{Abstract}

Rapidly destructive osteoarthritis (RDOA) is considered a rare and poorly diagnosed disease. RDOA is a destructive arthropathy that occurs most commonly in elderly women. The most typical radiological features are narrowing of the articular cartilage (chondrolysis) subchondral bone fracture, cysts in the femoral head/acetabulum, absence of osteophytes, flat femoral heads, and signs of joint effusion. RDOA was originally described in the hip but may also involve the shoulder and the knee. The pathogenesis of RPOA is not well understood, but subchondral fracture probably plays a role in the development of destruction of the joint. Nevertheless, early diagnosis of the patients with rapid destructive arthritis of the hip is important to try to reduce complexity of surgical intervention.

Key words: rapidly destructive osteoarthritis, hip, shoulder, knee, MRI, chondrolysis, subchondral fracture, pathogenesis 


\section{Introduction}

L'arthrose destructrice rapidement progressive (ADRP) est considérée comme une entité rare ; cette ADRP a d'abord été décrite à la hanche en 1970 par Lequesne et al., définie radiologiquement par un pincement de l'interligne articulaire d'au moins deux millimètres $(\mathrm{mm})$ par an ou de plus de $50 \%$ en un an, soit une disparition complète de l'interligne en 24 mois [1] ; cette arthropathie est remarquable par la vitesse de destruction du cartilage de l'articulation coxofémorale et l'absence de signes de reconstruction, en particulier l'absence d'ostéophytes ; elle a ensuite été décrite à l'épaule, au genou et à d'autres articulations.

L'aspect clinique et radiologique peut porter à confusion, car l'infection, les rhumatismes inflammatoires, les arthropathies métaboliques (ochronose, maladie de Wilson, hémochromatose, arthropathie amyloïde des dialysés) et nerveuses, l'ostéonécrose peuvent aussi se traduire par des arthropathies rapidement destructrices. Le diagnostic précoce est difficile car la symptomatologie clinique et la biologie sont non spécifiques et le handicap modéré. Cependant l'ADRP a une certaine spécificité clinique et radiologique qui permet d'orienter le diagnostic. L'IRM dans des stades précoces pourrait prédire les lésions destructrices ultérieures [2].

\section{Arguments cliniques}

\subsection{Hanche}

La coxarthrose destructrice rapide (CDR) est une forme clinique de coxarthrose avec une vitesse de chondrolyse plus rapide $(2 \mathrm{~mm} / \mathrm{an})$ que la coxarthrose semi-rapide ( $1 \mathrm{~mm} / \mathrm{an})$ et la coxarthrose commune $(0,25 \mathrm{~mm} / \mathrm{an})$; elle constitue 3 à $10 \%$ des coxarthroses, survenant sur une hanche indemne ou dégénérative [3].

La CDR prédomine chez la femme (60-90\%), plutôt âgée (âge moyen de 72 ans vs 61 ans chez l'homme, jamais avant 45 ans), de topographie unilatérale (80$90 \%$ ), parfois bilatérale $[1,2,4]$. Les facteurs de risque, en dehors de l'âge et du sexe féminin, sont l'obésité, la surcharge mécanique, les traumatismes de type indirect (chute, grand écart) ou direct (fracture acétabulaire rencontrée dans $6 \%$ des ADRP) $[2,3,5]$.

La symptomatologie douloureuse survient rapidement (2 à 16 mois avant l'apparition des lésions destructrices) avec impotence sévère ; les douleurs sont de rythme mécanique évoluant depuis plusieurs mois, émaillées d'épisodes de rythme inflammatoire, contrastant avec des mobilités souvent conservées, notamment en 
flexion ; l'évolution est marquée par l'apparition d'une boiterie et d'une limitation de la mobilité ; la modification brutale du rythme des douleurs et l'intensité des douleurs doivent attirer l'attention, bien qu'il puisse s'agir d'une poussée inflammatoire [2-4]. 2.2 Épaule

L'ADRP de l'épaule a été décrite par Lequesne et al. en 1982 [6], chez 6 femmes, avec des signes cliniques et radiologiques similaires à ceux de la CDR, comportant une ostéolyse (24 à $100 \%$ ) de la surface articulaire sur une période de 6 mois et une rupture de la coiffe des rotateurs. Le signe majeur est le collapsus de la tête humérale qui peut survenir en 6 mois, parfois en un mois $[2,7,8]$.

\section{$2.3 \mathrm{Genou}$}

La chondrolyse rapide peut aussi atteindre le genou ; il s'agit de la gonarthrose destructrice rapide (GDR), qui peut être idiopathique ou survenir notamment après une méniscectomie sous arthroscopie [9]. La GDR pourrait être définie par une perte du cartilage fémoro-tibial d'au moins $50 \%$ en un an par analogie avec la définition de Lequesne pour l'articulation de la hanche, avec une incidence de 5,8\%.

\subsubsection{Profil de la gonarthrose destructrice rapide (GDR)}

Le profil de la GDR a été précisé par une étude rétrospective portant sur 111 patients [10] : survenue chez des patients âgés en moyenne de 64 ans, avec un léger surpoids, prédominance féminine, atteinte du compartiment médial dans $79,3 \%$ des cas ; la sévérité de la douleur et de l'impotence fonctionnelle était variable. L'épanchement chronique mécanique du genou résistant aux infiltrations de corticoïdes était présent dans $91,9 \%$ des cas lors de la ponction, ce qui doit attirer l'attention, car l'importance du pincement de l'interligne articulaire à un an était corrélée au nombre de poussées de la maladie et au nombre de ponctions de liquide synovial durant l'année de suivi [10].

2.3.2 Données de la cohorte OAI (OsteoArthritis Initiative)

La cohorte prospective longitudinale (4 ans de suivi) OAl (OsteoArthritis Initiative) a permis de :

- préciser les facteurs prédictifs d'un remplacement prothétique du genou qui étaient cliniques : antécédents de chirurgie du genou (RR 1,$06 ; 1,02-1,11$ ), flexum (en degré) $(1,06 ; 1,02-1,01)$, EVA douleur, flexion active $(1,58 ; 1,04-$ 2,29) [11] ;

- retenir comme facteur d'accélération de la dégradation du cartilage, définie par une évolution vers les stades 3-4 de Kelgren-Lawrence $(K L)$ : 
- le traumatisme du genou survenu pendant la période d'observation (OR $3,14)$, avec une dégradation très rapide $(<1$ an) si le traumatisme était récent (OR 8,46), surtout chez les sujets les plus âgés [12] ;

- des lésions de l'os sous-chondral ou compromettant la fonction du ménisque (IRM) qu'il s'agisse d'extrusion, de fissures ou de modifications de forme, permettant d'identifier les sujets à risque [13] ;

- préciser les facteurs de risque dans les 2 à 4 ans précédant le début des signes radiographiques d'une gonarthrose d'évolution accélérée (passage du KL de 0 à 4 en moins de 4 ans), à savoir l'âge avancé, l'IMC élevé, les troubles statiques, l'arthrose controlatérale, la symptomatologie (douleur, handicap, épanchement, modifications du tissu adipeux de Hoffa, anomalies IRM ostéoarticulaires) [14].

2.3.3 Influence des cristaux de pyrophosphate de calcium sur la dégradation du cartilage

La méniscectomie, la chondrocalcinose et l'ostéonécrose semblent pouvoir induire une destruction rapide du cartilage [10].

Les données concernant l'influence des cristaux de pyrophosphate de calcium sur l'accélération de la dégradation cartilagineuse sont cependant contradictoires ; la réponse est oui selon l'étude des 70 sujets (67 ans en moyenne, $56 \%$ de femmes) avec une chondrocalcinose du genou droit (radio, IRM) et des signes d'arthrose modérée (Kellgren-Lawrence $<2$ ), suivis 4 ans, issus de la cohorte longitudinale Osteoarthritis Initiative [15] ; la réponse est non selon l'étude de 656 patients (62 ans en moyenne, $70 \%$ de femmes) avec une gonarthrose symptomatique (93 avec une chondrocalcinose), issus de la cohorte française KHOALA ; le critère principal était le risque de remplacement prothétique à 5 ans qui n'était pas influencé, tout comme la progression de l'arthrose, par la présence d'une chondrocalcinose [16].

L'arthrose destructrice rapide de l'articulation fémoro-patellaire est moins connue, pouvant survenir après une amyotrophie sévère et rapide de la cuisse dans une observation, considérée comme une cause de chondrolyse rapide de l'articulation fémoro-patellaire, ce qui démontre le rôle important de la force musculaire dans le développement de l'arthrose fémoro-patellaire [17].

\section{Arguments biologiques}


La CRP (C Reactive Protein) et la VS (Vitesse de Sédimentation) sont normales, parfois discrètement augmentées (20-30\%) [3], sachant qu'il peut exister une inflammation de bas grade dans le processus arthrosique [18].

L'intérêt des marqueurs cartilagineux et osseux est discuté. Les taux de TRACP-5b (tartrate-resistant acid phosphatase 5b) [19] et de MMP-3 (matrix metalloproteinase 3) sont plus élevés dans l'ADRP que dans l'arthrose et l'ostéonécrose ; ce haut niveau de remodelage osseux pourrait refléter l'activation des cellules ostéoclastiques dans l'ADRP [20], expliquant la destruction de la tête fémorale dans le stade précoce de l'ADRP [21]. Le taux de MMP-3 pourrait prédire la survenue de cette destruction de la tête fémorale [22].

L'examen du liquide synovial est utile quand il est possible, avec comptage cellulaire, recherche de microcristaux et examen bactériologique. Dans la CDR, le liquide est de type mécanique [3] ; à l'épaule, on peut mettre en évidence des cristaux de phosphate de calcium basique et parfois de pyrophosphate de calcium [7] ou de cholestérol [23] (diagnostic différentiel).

L'examen histologique n'est souvent fait qu'à un stade avancé, pouvant montrer une synoviale siège de débris ostéo-cartilagineux, hyperplasique, hypervascularisée, infiltrée par des fibroblastes, des histiocytes, des macrophages, des ostéoclastes matures, des cellules géantes et une fibrose capsulaire ; des lésions chondrales débordant les zones de contrainte, parfois une fracture sous-chondrale et un remaniement de l'os sous-chondral (fragmentation et régénération de la matrice osseuse, haut niveau de remodelage) avec des petites zones de nécrose non limitées [2-4].

\section{Arguments radiologiques}

L'imagerie reflète la surcharge mécanique, la réaction inflammatoire synoviale et osseuse, et la réponse osseuse favorable ou non favorable.

La radiographie initiale peut être considérée comme normale, et seule la répétition des clichés permettra de détecter la chondrolyse, surtout en cas de poussées inflammatoires itératives. L'aspect radiologique standard ne permet pas toujours la détection de zones de chondrolyse qu'on voit à l'arthroscopie ou sur l'arthroscanner. La chondrolyse rapide est suivie d'une résorption et d'une destruction osseuse sous-chondrale rapide et marquée [2].

\subsection{Hanche}


Les radiographies de hanche doivent être répétées tous les 2-3 mois (face et faux profil de Lequesne) si la douleur est sévère et durable, et ce d'autant que la coxarthrose est peu évoluée.

\subsubsection{Stade de chondrolyse rapide}

Le pincement de l'interligne articulaire coxo-fémoral est asymétrique, supérieur ou supéro-externe, accompagné d'une réaction ostéophytique minime, d'une condensation sous-chondrale faible, parfois de géodes sous-chondrales, achevé en 1 à 2 ans (Fig. 1,2) [2,4].

L'IRM dans des stades précoces pourrait prédire les lésions destructrices ultérieures : perte du cartilage, synovite, épanchement ; œdème extra-articulaire (confusion avec sepsis), notamment musculaire [24] ; œdème de la moelle osseuse de la tête fémorale et de l'acétabulum [25] (Fig. 3AB) [2].

La mise en évidence d'une fracture sous chondrale focale est importante dans les stades précoces [2] en cherchant sur I'IRM une bande curviligne en hyposignal (mieux visible en pondération T1) dans la zone de contrainte de l'os sous-chondral, parallèle à l'os sous-chondral et inférieur à $1 \mathrm{~cm}$ entourée d'œdème osseux diffus [25], permettant de faire la différence avec une ostéonécrose où la bande en hyposignal est concave vis-à-vis de la surface articulaire, sinueuse, circonscrivant la zone nécrotique (Fig 4).

Cette bande hypointense correspond à la fracture sous-chondrale et au processus de réparation (tissu nécrotique, fibrose de la moelle osseuse) ; elle est souvent irrégulière, discontinue, convexe vis-à-vis de la surface articulaire (Fig 4) ; des géodes sous-chondrales sont fréquemment associées [24-26].

\subsubsection{Stade tardif de destruction de l'os sous-chondral}

La tête fémorale est remaniée : elle s'ovalise, s'aplatit, se subluxe du cotyle siège d'une ostéolyse (1 mm/an) ; ce remaniement est parfois important évoquant une neuroarthropathie mais il n'y a pas de fragmentation et de débris osseux intraarticulaires ; le remodelage de l'acétabulum est variable (parfois intact) ; I'IRM donne ici peu d'information diagnostique (synovite et œdème de la moelle osseuse) [2-3].

\subsection{Epaule}

S'il existe une certaine similarité avec la hanche [8], il n'y a pas de séparation nette entre les 2 phases de chondrolyse et de résorption osseuse ; les caractéristiques communes sont l'aplatissement de la surface articulaire (collapsus de la tête humérale qui peut survenir en 6 mois, parfois en un mois [7,8]), interne ou supérointerne, associé 
à une condensation sous chondrale et une ostéophytose minime ; la destruction osseuse est parfois sévère (100 \%) ; l'aspect de la glène est variable, souvent préservée, parfois résorbée ; l'ostéolyse marquée et la destruction ostéoarticulaire majeure, l'érosion de la tête humérale évoquent une neuroarthropathie, ce d'autant que les douleurs sont modérées ; on peut aussi observer des débris osseux intraarticulaires, des calcifications dans la capsule articulaire distendue, une distension de la bourse sous-acromiale, et un épaississement de la coiffe des rotateurs (rupture transfixiante, dégénérescence graisseuse des muscles de la coiffe) différant cependant du tableau de la " cuff tear arthropathy " car il n' y a pas de migration proximale de la tête humérale et pas de rupture massive de la coiffe $[2,7,8]$.

Les données de l'IRM sont similaires à celles de la CDR : épanchement massif et synovite, œdème de la moelle osseuse péri-lésionnel (tête humérale et métaphyse) comme dans les lésions de nécrose, fracture sous-chondrale en pondération T1 et T2 ; l'aplatissement de la tête humérale et le remodelage de la tête sont identiques aux données de la radiographie standard $[2,7,8]$.

\subsection{Genou}

La répétition des radiographies en charge en extension et en schuss, dans le même centre de radiologie, en utilisant les mêmes procédures, est le moyen le plus efficace pour détecter le pincement rapide de l'interligne fémoro-tibial, sans ostéophytose associée [10].

\subsection{Autres localisations}

La rhizarthrose prend parfois l'allure d'une forme destructrice rapide où l'interligne articulaire se détruit en un à deux ans avec une destruction partielle de l'os trapèze, révélée par une douleur importante et une incapacité fonctionnelle rapidement installée [27].

L'arthrose digitale peut devenir érosive (ou inflammatoire), cliniquement inflammatoire et radiologiquement destructrice qui peut simuler de très près un rhumatisme psoriasique digital ou l'arthropathie destructrice des doigts de la chondrocalcinose articulaire [28].

L'articulation radiocarpienne se détruit parfois après arthroscopie [29].

\section{Pathogénie}


De nombreuses hypothèses ont été évoquées pour expliquer l'ADRP, soit comme une pathologie unique mécanique ou métabolique, soit comme une voie finale de pathologies multiples et différentes [7].

\subsection{Surcharge mécanique}

La surcharge mécanique peut impacter le cartilage (hyperpression sur un cartilage normal ou anormal ou arthrosique) et l'os sous-chondral à type d'ischémie ou d'ostéonécrose primitive basée sur les analyses histologiques [8,30], notamment en cas d'inversion du labrum acétabulaire source de fracture sous-chondrale [31].

\subsection{Rôle des fractures sous-chondrales}

Le rôle des fractures sous-chondrales est argumenté par de nombreuses études pour la hanche et l'épaule. Concernant l'épaule, les lésions de la coiffe des rotateurs, entraînant une perturbation de la biomécanique, peuvent être source d'une fracture sous-chondrale ; celle-ci est-elle la cause de la chondrolyse et de la destruction osseuse ou la conséquence d'un autre processus ? Certaines fractures sous-chondrales se compliquent d'ADRP mais pas toutes [2,8,32]. De la même façon, la mise en évidence de fractures sous-chondrales à l'origine de certaines CDR corrobore l'hypothèse de certains auteurs selon laquelle l'os sous-chondral et ses microfractures sont des acteurs importants dans la pathogénie de l'ADRP [3,33]. II ne s'agit pas de fractures par insuffisance osseuse, car il n'existe pas de modifications de la densité minérale osseuse dans cette pathologie [2,4,34].

\subsection{Rôle des ostéoclastes}

Les ostéoclastes jouent un rôle important dans la résorption osseuse, présents dans la synoviale, dans le tissu de granulation hypervascularisé et sont plus nombreux dans l'ADRP que dans l'arthrose commune, notamment dans les zones actives ; la CDR est associée à une augmentation du taux du taux des molécules favorisant la résorption osseuse (IL-6, IL-1 $\beta$, métalloprotéinases). L'endothélium pourrait jouer un rôle important dans le recrutement des précurseurs des ostéoclastes sur les sites de résorption [5].

\subsection{Rôle de la membrane synoviale}

L'inflammation de la membrane synoviale, secondaire à la présence de débris ostéocartilagineux, entretient la chondrolyse (prostaglandines, cytokines, métalloprotéases) et entraîne des phénomènes douloureux. Toutes les cytokines mesurées dans l'ADRP sont à un taux plus élevé que dans l'arthrose, et notamment I'IL-8, témoin de l'agressivité de la destruction articulaire [35]. 


\subsection{Rôle des cristaux}

L'inflammation peut aussi être induite par les cristaux (pyrophosphate de calcium ou PPCa et phosphate de calcium basique ou PCB). En particulier, les cristaux de PCB peuvent entraîner une inflammation synoviale et une dégradation du cartilage, suggérant que ces cristaux ont un rôle pathogénique direct dans l'arthrose ; ces effets sont indépendants de l'IL-1 et de l'inflammasome NLRP3 [36]. On rappelle que l'association des cristaux de PCB avec un type particulier d'arthropathie dégénérative ou destructrice de l'épaule, appelée épaule de Milwaukee, est en faveur de leur rôle pathogène possible dans l'arthrose [2,37]. Les nouvelles techniques d'imagerie jouent un rôle important dans l'amélioration des connaissances et de la compréhension du rôle pathogène éventuel de divers cristaux de calcium dans les articulations in vivo, notamment dans l'arthrose [38].

\subsection{Rôle des médicaments}

\subsubsection{Antagonistes du NGF (nerve growth factor)}

Certains médicaments peuvent être inducteurs d'arthropathie destructrice, comme les antalgiques type indométacine [39] et les antagonistes du nerve growth factor (NGF), sujet éminemment d'actualité [40].

On rappelle que la FDA a suspendu an avril 2010 les essais cliniques du tanezumab dans les affections non cancéreuses, en raison de l'apparition non rare (3 pour 100 patients -année) d'arthropathies (étiquetées ostéonécroses) nécessitant un remplacement prothétique, suspension étendue à tous les anti-NGF en décembre 2010. Une commission (Arthritis Advisory Committee ou AAC), mandaté par la FDA, a examiné les dossiers de 353 patients souffrant de 500 arthropathies invalidantes, dont $80 \%$ étaient rapportées à l'évolution progressive normale de l'arthrose, les autres se manifestant sous forme d'arthropathies destructrices rapides pour lesquelles les antiNGF pouvaient être incriminées (atteinte d'articulations non portantes ou indemnes d'arthrose, relation dose-effet, durée d'utilisation, rôle aggravant des AINS associés). Des études expérimentales complémentaires ont été demandées par l'AAC et l'interdiction des essais a été levée en juillet 2013 [40].

Le sponsor du tanézumab (Pfizer) a mis sur pied un comité d'adjudication dont le rapport a été présenté à une réunion de la FDA en mars 2012 et à l'ACR de la même année, résumé par Hochberg [33,41] ; 162 patients ayant eu un remplacement prothétique ont été retenus pour l'adjudication et des effets indésirables 
ostéoarticulaires ont été décrits par les investigateurs sous la dénomination " ostéonécroses » chez 87 patients durant les études de phase II/III, touchant la hanche $(54,7 \%)$, le genou (30,5\%) et l'épaule (12,6\%); à la fois le nombre de cas et le taux par 1000 personnes-année d'exposition étaient plus élevés dans le groupe randomisé comprenant l'association tanezumab-AINS.

Les cas ont été classés par les experts en ostéonécrose primitive (seulement 2 cas), arthrose rapidement progressive ( $\mathrm{ARP}^{\circ}$ ) de type 1 (pincement de l'interligne $\geq 1$ ou $2 \mathrm{~mm}$ en moins d'un an, sans lésion osseuse majeure, arthrose rapidement progressive (ARP) de type 2 (perte ou destruction osseuse non habituellement présente dans l'arthrose évoluée, sans réaction ostéophytique), progression normale de l'arthrose ; les résultats concernant les 87 patients sont détaillés dans le Tableau 1. Le mécanisme de ces ARP n'est pas parfaitement connu : lésions nerveuses (peu probable), surutilisation des articulations portantes en raison de l'analgésie, à l'origine d'une surcharge mécanique, rôle des prostaglandines (thrombose microvasculaire osseuse), fractures sous-chondrales (expliquant la perte osseuse) dont la réparation peut être inhibée par les AINS [33].

Actuellement, deux molécules sont désormais en phase 3 , le tanezumab (laboratoires PFIZER et LILLY) et le fasinumab (laboratoires REGENERON et TEVA), tous deux par voie d'administration sous-cutanée (2,5 et $5 \mathrm{mg}$ toutes les 8 semaines pour le tanezumab et $1 \mathrm{mg}$ toutes les 4 ou 8 semaines pour le fasinumab). Ces études de grande ampleur, ont pour objectif essentiel d'évaluer la bonne tolérance du traitement et en particulier de s'assurer que le plan de réduction des risques, (à savoir une dose plus faible administrée comparativement aux premiers essais et la contreindication à l'association à un AINS) est bien efficace [42]. Des résultats viennent d'être publiés pour ces 2 molécules [43-45] ; il y a une nette diminution des effets indésirables ostéoarticulaires comparée aux données du rapport du comité d'adjudication, notamment pour l'ARP de type 2 (Tableau 1).

\subsubsection{Corticoïdes intraarticulaires}

Les effets des injections intraarticulaires de corticoïdes sont discutés. L'infiltration intra-articulaire d'un corticoïde, suivie ou non d'une mise en décharge, ne permet pas d'éviter la mise en place d'une prothèse totale de hanche au cours de la CDR [46]. Dans une série de 100 patients (129 injections intra-articulaires de corticoïdes dans la hanche), 23 cas (21\%) de CDR ont été répertoriés [47]. 


\section{Diagnostic}

\subsection{Diagnostic positif}

Le diagnostic d'ADRP est basé sur des arguments anamnestiques, cliniques, biologiques (importance de l'analyse du liquide synovial), radiologiques et évolutifs ; le diagnostic précoce reste difficile car sa survenue est difficilement anticipable et le diagnostic est généralement posé une fois la destruction effectuée. Cependant, l'ADRP a une certaine spécificité clinique et radiologique qui permet d'orienter le diagnostic et d'éliminer les arthroses secondaires [48].

L'interrogatoire doit rechercher la notion de consommation d'alcool et la prise de corticoïdes [2]. L'examen clinique est important si l'on veut écarter une neuroarthropathie [2], notamment à l'épaule (Fig. 5) ou à la hanche (Fig. 6) représentant $5 \%$ des cas (diabète, tabès, syringomyélie) [49], ce d'autant que la sémiologie clinique est non spécifique, que la douleur est le symptôme révélateur (paradoxal pour une neuroarthopathie habituellement indolore) et que l'atteinte structurale évolue rapidement.

L'imagerie doit être interprétée avec attention.

À la hanche, la coexistence d'une douleur intense (disproportionnée) avec impotence fonctionnelle majeure, contrastant avec une mobilité passive encore peu limitée [1], nécessite une radiographie, une échographie et une CRP (Fig. 7).

a) La radiographies standard peut-être normale au début mais est importante pour être la référence lors du suivi radiographique (tous les 2-3 mois) [1] et permet d'éliminer les principaux diagnostics différentiels (chondrocalcinose, lésion osseuse fissuraire, tumorale pour une fracture pathologique et ostéonécrose aseptique). L'échographie $[1,50]$ permet de confirmer la présence d'un épanchement (quasi constant à la hanche initialement), de le ponctionner pour éliminer une arthrite (éléments nucléés $>2000 / \mathrm{mm}^{3}$ ) septique (bactérie) ou métabolique (cristaux) et de rechercher des vésicules lipidiques pour une ostéonécrose aseptique de la tête fémorale. La CRP doit être inférieure à $10 \mathrm{mg} / \mathrm{L}$ (la vitesse de sédimentation peut être un peu augmentée dans 20 à $30 \%$ des CDR) [1,50].

b) En cas de radiographie sub-normale, avec un liquide mécanique, il faut faire une IRM pour éliminer une ostéonécrose aseptique de la tête fémorale de stade 1, rechercher une fracture sous chondrale et un œdème extensif sous chondral avec éventuellement atteinte des parties molles qui contraste avec l'absence de germe et 
le liquide mécanique qui fait qu'il n'y a plus vraiment d'autre diagnostic différentiel (sauf peut-être l'ostéome ostéoïde) que la CDR.

\subsection{Diagnostic différentiel}

II faut éliminer les arthropathies inflammatoires, infectieuses, nerveuses, en particulier tabétique (Fig. 6), les arthropathies métaboliques (ochronose, maladie de Wilson, hémochromatose, arthropathie amyloïde des dialysés), ainsi que les rares chondrolyses multifocales récemment décrites chez les patients ayant eu une greffe hépatique ; les arthropathies microcristallines (PPCa, PCB) et l'ostéonécrose aseptique (nécrose avec chondrolyse rapide ou coxopathie ischémique) [30] peuvent aussi s'accompagner d'ADRP [3] ; mais les éléments anamnestiques, cliniques, biologiques et radiologiques de ces entités sont bien différents [7].

L'ADRP de l'épaule doit être distinguée [7] : de l'épaule sénile hémorragique (Fig. 8), de l'épaule de Milwaukee (débris osseux pulvérisés dans l'espace articulaire avec présence de cristaux d'apatite) [51], et de la « cuff tear arthropathy » (rupture importante de la coiffe des rotateurs entraînant une dégradation de l'articulation glénohumérale puis un collapsus sous chondral déformant la tête et libérant des fragments articulaires au sein de la membrane synoviale) [52].Toutes ces entités, regroupées sous la dénomination d'arthropathie destructrice rapide de l'épaule, ont des caractéristiques communes : le terrain (patients âgés, prédominance féminine), la douleur modérée à début insidieux et la limitation de la mobilité après rupture de la coiffe des rotateurs, l'instabilité articulaire (volumineux épanchement teinté de sang), l'atteinte sévère dans $13 \%$ quand il existe des antécédents de CDR [2]. L'existence d'une déformation de la tête humérale qui perd sa sphéricité et s'aplatit, avec un interligne articulaire gléno-huméral qui reste au départ préservé peut orienter vers une ostéonécrose mais les autres structures articulaires, notamment la glène, ne sont pas impliquées, ce qui différencie nettement cette pathologie des arthropathies destructrices [7]. On a vu l'importance de I'IRM pour distinguer l'ADRP de l'ostéonécrose [24-25]. Les clichés de bassin, poignets et genoux sont utiles pour rechercher une chondrocalcinose.

\section{Traitement}

La prise en charge médicale se limite à la prescription d'antalgiques et d'AINS à visée symptomatique. Les bisphosphonates et la vitamine $D$ n'ont aucun effet sur I'ADRP [5]. 
La mise en décharge stricte proposée par certains auteurs, associée ou non à une injection d'un dérivé cortisoné, ne semble pas ralentir l'évolution de la CDR [46]. L'infiltration intra-articulaire de corticoïdes peut augmenter le risque infectieux périopératoire d'une PTH si elle est réalisée dans les 3 mois avant la chirurgie [53]. Si l'on prend en compte la fréquence de l'existence de fractures sous-chondrales, il est licite de diminuer les contraintes à la hanche à l'aide d'une paire de béquilles selon la technique du pas simulé pendant 3 à 4 semaines.

Le pronostic de la CDR est sévère, conduisant dans les 12 à 43 mois suivant les infiltrations de corticoïdes, avec ou sans mise en décharge par des béquilles, à un remplacement prothétique $(96,4 \%)$ [46].

Le seul traitement efficace est la chirurgie prothétique à proposer lorsque la douleur et la gêne fonctionnelle impactent significativement la qualité de vie du patient. La chirurgie peut être compliquée par un saignement plus abondant et des difficultés techniques dues à une perte osseuse acétabulaire importante, d'où l'intérêt d'intervenir avant l'apparition des dégâts acétabulaires. Les résultats sont bons, supérieurs à $95 \%$ à 5 ans [4-5].

Le pronostic de la GDR paraît moins sévère ; en effet, dans l'étude rétrospective de Burki et al. [10], portant sur 111 patients atteints de GDR, après une prise en charge médicale globale incluant le lavage articulaire suivi d'une infiltration de corticoïdes (pour évacuer les débris cartilagineux, les cytokines, les cristaux et diminuer la réaction synoviale) et combinant d'autres traitements pharmacologiques et non pharmacologiques, plus de la moitié des patients $(58,4 \%)$ n'avaient pas eu recours à la chirurgie après une période moyenne de suivi de 55,0 mois, avec un état algofonctionnel acceptable permettant la reprise du travail chez les patients en activité. Ces traitements intra-articulaires doivent être suivis d'une décharge relative en conseillant la perte de poids, l'exercice musculaire et le port d'orthèses [10].

\section{Conclusions}

L'ADRP est une arthropathie destructrice survenant le plus souvent chez la femme âgée ou chez des sujets ayant une surcharge articulaire mécanique, traumatique ou non, touchant essentiellement la hanche, le genou et l'épaule. La mise en évidence chez un patient d'un pincement de l'interligne articulaire évolutif doit conduire à un bilan visant à apprécier la perte et la destruction osseuse. L'IRM dans 
des stades précoces pourrait prédire les lésions destructrices ultérieures ; il faut particulièrement rechercher un œdème de la moelle osseuse, une fracture et des géodes sous-chondrales, ce qui permet d'identifier les patients à risque de destruction osseuse. Le seul traitement est le remplacement prothétique à effectuer avant l'apparition des dégâts acétabulaires. 


\section{Références}

1. Lequesne M, Sèze $S$ de, Amouroux J. La coxarthrose destructrice rapide. Rev Rhum Mal Ostéoartic 1970;37:721-33.

2. Flemming DJ, Gustas-French CN. Rapidly Progressive Osteoarthritis: a Review of the Clinical and Radiologic Presentation. Curr Rheumatol Rep 2017;19:42.

3. Richette P. La coxarthrose destructrice rapide. Rev Rhum 2009;76:341-5.

4. Mavrogenis AF, Flevas DA, Panagopoulos GN, Megaloikonomos P, Igoumenou V, Vottis C, et al. Rapid destructive arthritis of the hip revisited. Eur J Orthop Surg Traumatol 2015;25:1115-20.

5. Hu L, Zhang $X$, Kourkoumelis N, Shang $X$. The mysteries of rapidly destructive arthrosis of the hip joint: a systemic literature review. Ann Palliat Med 2020;9:1220-9.

6. Lequesne M, Fallut M, Coulomb R, Magnet JL, Strauss J. L'arthropathie destructrice rapide de l'épaule. Rev Rhum 1982;49:427-37.

7. Thomas T. L'arthropathie destructrice de l'épaule. Rev Rhum Monogr 2018;85:134-7.

8. Kekatpure AL, Sun JH, Sim GB, Chun JM, Jeon IH. Rapidly destructive arthrosis of the shoulder joints: radiographic, magnetic resonance imaging, and histopathologic findings. J Shoulder Elbow Surg 2015;24:922-7.

9. Steinmetz S, Bonnomet F, Rahme M, Adam P, Ehlinger M. Rapid chondrolysis of the medial knee compartment after arthroscopic meniscal resection: a case report. $J$ Med Case Rep 2016;10:81.

10. Burki V, Paternotte S, Dougados M, Ayral X. Arthrose fémoro-tibiale rapidement destructrice : présentation clinico-radiologique et devenir après traitement médical global incluant le lavage articulaire non arthroscopique associé à une infiltration de corticoïdes. Étude rétrospective monocentrique. Rev Rhum 2014;81:74-80.

11. Riddle DL, Kong X, Jiranek WA. Factors associated with rapid progression to knee arthroplasty: complete analysis of three-year data from the osteoarthritis initiative. Joint Bone Spine 2012;79:298-303.

12. Driban JB, Eaton CB, Lo GH, Ward RJ, Lu B, McAlindon TE. Association of knee injuries with accelerated knee osteoarthritis progression: data from the Osteoarthritis Initiative. Arthritis Care Res 2014;66:1673-9.

13. Driban JB, Ward RJ, Eaton CB, Lo GH, Price LL, Lu B, McAlindon TE. Meniscal extrusion or subchondral damage characterize incident accelerated osteoarthritis:

Data from the Osteoarthritis Initiative. Clin Anat 2015;28:792-9.

14. Driban JB, Harkey MS, Barbe MF, Ward RJ, MacKay JW, Davis JE, et al. Risk factors and the natural history of accelerated knee osteoarthritis: a narrative review.

BMC Musculoskelet Disord 2020;21:332. 
15. Foreman SC, Gersing AS, von Schacky CE, Joseph GB, Neumann J, Lane NE, et al. Chondrocalcinosis is associated with increased knee joint degeneration over 4 years: data from the Osteoarthritis Initiative. Osteoarthritis Cartilage 2020;28:201-7.

16. Latourte A, Rat AC, Ngueyon Sime W, Ea HK, Bardin T, Mazières B, et al. Chondrocalcinosis of the Knee and the Risk of Osteoarthritis Progression: Data From the Knee and Hip Osteoarthritis Long-term Assessment Cohort. Arthritis Rheumatol 2020;72:726-32.

17. Ferrari M, Louati K, Miquel A, Behin A, Benveniste O, Sellam J. Amyotrophie rapidement progressive de la cuisse : un cas inhabituel de chondrolyse rapide du genou. Rev Rhum 2015;82:408-10.

18. Courties A, Berenbaum F, Sellam J. The Phenotypic Approach to Osteoarthritis: A Look at Metabolic Syndrome-Associated Osteoarthritis. Joint Bone Spine 2019;86:725-30.

19. Yamaguchi R, Yamamoto T, Motomura G, Ikemura S, Iwasaki K, Zhao G, et al. Bone and cartilage metabolism markers in synovial fluid of the hip joint with secondary osteoarthritis. Rheumatology 2014;53:2191-5.

20. Abe H, Sakai T, Ogawa T, Takao M, Nishii T, Nakamura N, Sugano N. Characteristics of bone turnover markers in rapidly destructive coxopathy. $\mathrm{J}$ Bone Miner Metab 2017;35:412-8.

21. Yasuda T, Matsunaga K, Hashimura T, Tsukamoto $Y$, Sueyoshi T, Ota S, et al. Bone turnover markers in the early stage of rapidly progressive osteoarthritis of the hip. Eur J Rheumatol 2020. doi: 10.5152/eurjrheum.2020.20046. [Epub ahead of print].

22. Yasuda T, Matsunaga K, Hashimura T, Tsukamoto Y, Sueyoshi T, Ota S, et al. Characterization of rapidly progressive osteoarthritis of the hip in its early stage. Eur J Rheumatol 2020;7:130-4.

23. Robin F, Cadiou S, Berthoud O, Bart G, Albert JD, Coiffier G. Thick and yellowish articular effusion: a challenging diagnosis! Focus on cholesterol crystal deposition in joint. Inflamm Res 2020;69:713-4.

24. Viana SL, Machado BB, Mendlovitz PS. MRI of subchondral fractures: a review. Skeletal Radiol 2014;43:1515-27.

25. Boutry N, Paul C, Leroy X, Fredoux D, Migaud H, Cotten A. Rapidly destructive osteoarthritis of the hip: MR imaging findings. Am J Roentgenol 2002;179:657-63.

26. Ikemura S, Mawatari T, Matsui G, Iguchi T, Mitsuyasu $H$. The depth of the lowintensity band on the T1-weighted MR image is useful for distinguishing subchondral insufficiency fracture from osteonecrosis of the collapsed femoral head. Arch Orthop Trauma Surg 2018;138:1053-8.

27. Chevalier X, Compaore C, Eymard F, Flipo RM. Rhizarthrose. Rev Rhum Monogr 2012;79:110-5. 
28. Chicheportiche V, Laredo JD. Diagnostic radiographique d'un rhumatisme des mains set des poignets. Rev Rhum Monogr 2012;79:165-72.

29. Shim E, Kim BH, Choi IY, Hong SJ, Kang CH, Ahn KS. Imaging appearance of post-arthroscopic radiocarpal chondrolysis: comparison with osteoarthritis associated with scapholunate dissociation. Acta Radiol 2020. doi: 10.1177/0284185120922819. [Epub ahead of print].

30. Laroche M, Moineuse C, Durroux R, Mazières B, Puget J. La coxopathie ischémique, une cause de coxarthrose des tructrice rapide ? A propos d'une observation. Rev Rhum [Ed Fr] 2002;69:84-9.

31. Fukui K, Kaneuji A, Fukushima M, Matsumoto T. Early MRI and intraoperative findings in rapidly destructive osteoarthritis of the hip: A case report. Int J Surg Case Rep 2015;8C:13-7.

32. Kakutani R, Kondo N, Mochizuki T, Fujisawa J, Endo N. Rapidly Destructive Arthrosis of Bilateral Humeral Heads Caused by Subchondral Insufficiency Fracture. Acta Med Okayama 2018;72:525-30.

33. Hochberg MC, Tive LA, Abramson SB, Vignon E, Verburg KM, West CR, et al. When Is Osteonecrosis Not Osteonecrosis?: Adjudication of Reported Serious Adverse Joint Events in the Tanezumab Clinical Development Program. Arthritis Rheumatol 2016;68:382-91.

34. Richette P, Vicaut E, de Vernejoul MC, Orcel P, Bardin T. Bone mineral density in patients with rapidly destructive or common hip osteoarthritis. Clin Exp Rheumatol 2009;27:337-9.

35. Abe H, Sakai T, Ando W, Takao M, Nishii T, Nakamura N, et al. Synovial joint fluid cytokine levels in hip disease. Rheumatology 2014;53:165-72.

36. Ea HK, Chobaz V, Nguyen C, Nasi S, van Lent P, Daudon M, et al. Pathogenic role of basic calcium phosphate crystals in destructive arthropathies. PLoS One 2013;8:e57352.

37. MacMullan P, McMahon G, McCarthy G. Détection des cristaux de phosphate de calcium basique dans l'arthrose. Rev Rhum 2011;78:220-6.

38. Becce F, Viry A, Stamp LK, Pascart T, Budzik JF, MARS Collaboration, Raja A. Du nouveau dans l'imagerie des maladies à dépôts de cristaux de calcium. Rev Rhum 2020;87:235-8.

39. Huskisson EC, Berry H, Gishen P, Jubb RW, Whitehead J. Effects of antiinflammatory drugs on the progression of osteoarthritis of the knee. LINK Study Group. Longitudinal Investigation of Nonsteroidal Antiinflammatory Drugs in Knee Osteoarthritis. J Rheumatol 1995;22:1941e6.

40. Bannwarth $B$, Kostine $M$. Antagonistes du nerve growth factor (NGF) : des antalgiques d'avenir ? Rev Rhum 2015;82:25-8. 
41. Hochberg MC. Serious joint-related adverse events in randomized controlled trials of anti-nerve growth factor monoclonal antibodies. Osteoarthritis Cartilage 2015;23(Suppl 1):S18-21.

42. Berenbaum F. Les anti-NGF dans l'arthrose : que faut-il en attendre ? Rev Rhum 2019;86:5-7.

43. Berenbaum F, Blanco FJ, Guermazi A, Miki K, Yamabe T, Viktrup L, et al. Subcutaneous tanezumab for osteoarthritis of the hip or knee: efficacy and safety results from a 24-week randomised phase III study with a 24-week follow-up period. Ann Rheum Dis 2020;79:800-10.

44. Schnitzer TJ, Easton R, Pang S, Levinson DJ, Pixton G, Viktrup L, et al. Effect of Tanezumab on Joint Pain, Physical Function, and Patient Global Assessment of Osteoarthritis Among Patients With Osteoarthritis of the Hip or Knee: A Randomized Clinical Trial. JAMA 2019;322:37-48.

45. Dakin P, DiMartino SJ, Gao H, Maloney J, Kivitz AJ, Schnitzer TJ, et al. The Efficacy, Tolerability, and Joint Safety of Fasinumab in Osteoarthritis Pain: A Phase Ilb/llI Double-Blind, Placebo-Controlled, Randomized Clinical Trial. Arthritis Rheumatol 2019;71:1824-34.

46. Villoutreix C, Pham T, Tubach F, Dougados M, Ayral X. Les infiltrations de corticoïdes dans la coxarthrose destructrice rapide. Rev Rhum 2006;73: 62-7.

47. Hess SR, O'Connell RS, Bednarz CP, Waligora AC 4th, Golladay GJ, Jiranek WA. Association of rapidly destructive osteoarthritis of the hip with intra-articular steroid injections. Arthroplast Today 2018;4:205-9.

48. Eymard F, Chevalier X. Fausses arthroses primaires. Rev Rhum Monogr 2018;85:295-301.

49. Floyd W, Lovell W, King RE. The neuropathic joint. South Med J 1959;52:563-9.

50. Lequesne M. Coxopathies chroniques de l'adulte : diagnostic et traitement. EMC - Appareil locomoteur 2000;1-21 [Article14-308-A-10].

51. 8. Ersoy H, Pomeranz SJ. Milwaukee Shoulder Syndrome. J Surg Orthop Adv 2017;26:54-7.

52. Rugg CM, Gallo RA, Craig EV, Feeley BT. The pathogenesis and management of cuff tear arthropathy. J Shoulder Elbow Surg 2018;27:2271-83.

53. Werner BC, Cancienne JM, Browne JA. The Timing of Total Hip Arthroplasty After Intraarticular Hip Injection Affects Postoperative Infection Risk. J Arthroplasty 2016;31:820-3. 
Tableau 1. Effets ostéoarticulaires indésirables pour le Tanézumab (TNZ) et le Fasinumab (FAS).

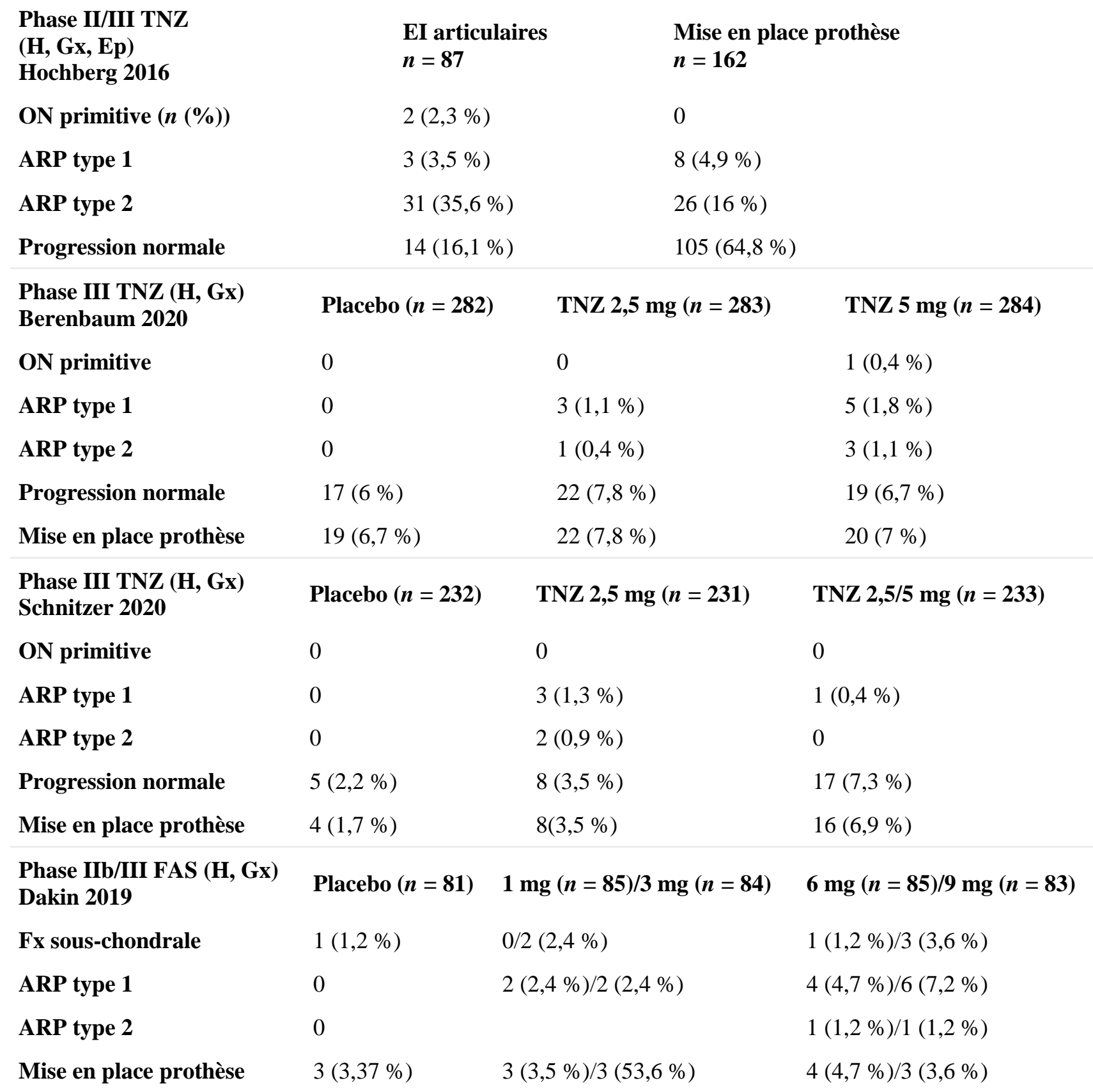

ARP : arthrose rapidement destructrice ; Ep : épaule ; EI : effets indésirables ; Fx : fractures ; Gx : genou ; $\mathrm{H}$ : hanche ; ON : ostéonécrose. 


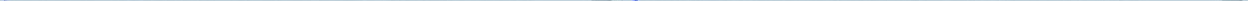




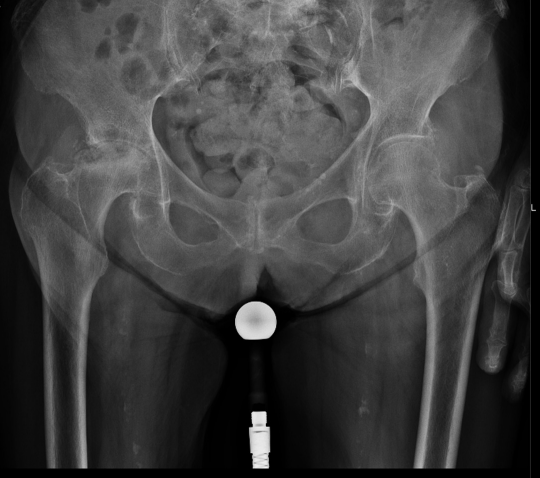


A

B 
Droit couché

○

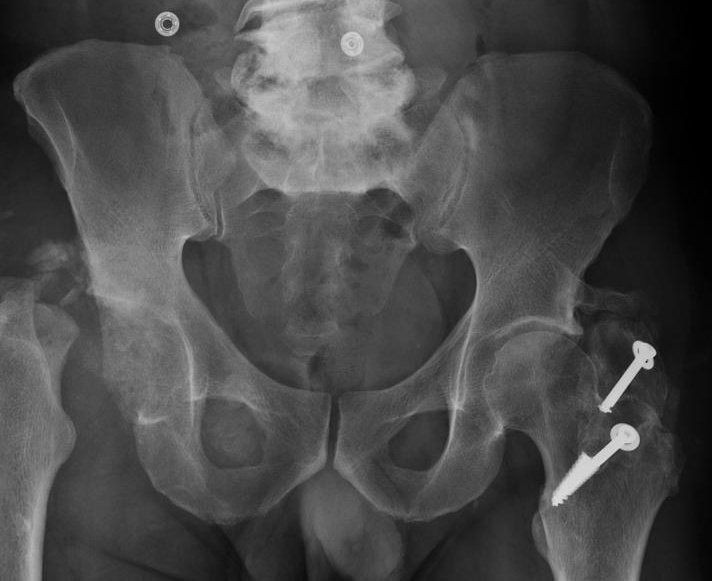




\section{Douleur de hanche intense avec impotence fonctionnelle majeure}

Radiographie + échographie + CRP

Parfois normale
Référence lors du suivi (tous les 2-3 mois)
Intérêt pour le diagnostic différentiel
(CCA, lésions osseuses)

\section{Echographie}

Confirme la présence d'un épanchement Analyse du liquide synovial pour éliminer une arthrite septique et métabolique
CRP

$<10$ $\mathrm{mg} / \mathrm{L}$

Radiographie subnormale avec liquide synovial mécanique (éléments nuclées $<1000$ éléments $/ \mathrm{mm} 3$ et CRP $<10 \mathrm{mg} / \mathrm{L}$

IRM

Éliminer l'ostéonécrose Rechercher éléments ADRP

- fracture sous-chondrale

- CEdème extensif

Antalgiques

Paire de cannes

anglaises

(pas simulé) 


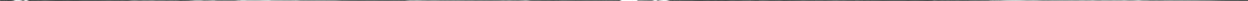

